\title{
Detection of Weak Signal Based on Parameter Identification of Delay Differential System with Noise Disturbance
}

\author{
QiuBao Wang (iD, Yuejuan Yang, and Xing Zhang \\ Department of Mathematical and Physics Shijiazhuang Tiedao University, Shijiazhuang 050043, Hebei, China \\ Correspondence should be addressed to QiuBao Wang; wangqiubao12@sina.com
}

Received 28 November 2019; Accepted 30 March 2020; Published 14 April 2020

Academic Editor: Dimitrios Mitsotakis

Copyright ( 2020 QiuBao Wang et al. This is an open access article distributed under the Creative Commons Attribution License, which permits unrestricted use, distribution, and reproduction in any medium, provided the original work is properly cited.

\begin{abstract}
Extraction of the weak signals is crucial for fault prognostics in which case features are often very weak and masked by noise. In the time domain, the detection of weak signals depends on the identification of nonlinear parameters. A new signal detection and estimation method based on the incremental harmonic balance (IHB) is developed from the stochastic Van der Pol-Duffing equation with delayed feedback under parametric excitation. This is the first time that the IHB has been applied for the identification of parameters in stochastic delay differential equations (SDDEs). Compared to the method of intermittency transition between order and chaos to detect weak signals, this new method is more direct and the calculation result is what we want to obtain. This new method is suitable for the generalization and application of SDDEs.
\end{abstract}

\section{Introduction}

It is well known that a self-excited system can exhibit a remarkable complex dynamical behaviour. The earliest mathematical model has been proposed by Van der Pol to describe the vacuum tube circuit [1], and subsequently, many researchers have studied the complex phenomena observed from experiments and computer simulations in the fields of mechanical, electric, ecological, and chemical systems and so on. For rotating machinery, undesirable bifurcations, high-amplitude vibrations caused by resonance, quasiperiodic motion, and chaotic behaviour may occur and cause degradation or catastrophic failure of the system.

Bearing failure is one of the foremost causes of breakdowns in rotating machinery, and such failure can be catastrophic, resulting in huge economic losses. To prevent these kinds of failures from happening, various bearing condition monitoring techniques have been developed. Among them, vibration analysis has been used extensively due to its intrinsic advantage of revealing bearing failure [2-4]. Detecting the dangerous vibrations is very crucial for engineering science, and various papers have been dedicated for the control of resonantly forced systems in various applicative fields; Atay [5] studied the effect of delayed position feedback on the response of a Van der Pol oscillator, showing that the delay can change the amplitude of limit cycle oscillations or suppress them altogether. Lai and Leng [6] considered a two-dimensional Duffing oscillator and proposed the weak-signal detection approach based on the GPASR model. A filtering technique through chaotic regime-switching is proposed by Denis et al. [7]. The inherent deficiency of the measuring mechanism leads to a mass of various noises to the effective signal inevitably. The signal of a defective bearing is spread across a wide frequency band and hence can become masked by noise and low-frequency signals with ease [8]. Prognostics is achieved by detecting the failure and defect at its initial stage and alerting maintenance personnel before it develops into a sharp loss. Nevertheless, in the early failure stage, it is difficult to detect the weak rolling bearing failure signal.

To solve the problem of detecting effective weak failure signals under strong noise, numerous scholars have proposed various methods: (1) apply the transform to achieve a goal of signal demodulation. Pang et al. [9] present an improved Hilbert time transform (IHTT), and the wavelet transform has been used for signal demodulation [10]. (2) Construct filters based on noise type and application to conduct the denoising $[11,12]$. (3) Utilize the characteristics 
of chaos system which is immune to noise and sensitive to weak signals at the same time to detect the weak effective failure signals. Zhao [13] detects the weak signal submerged in strong noise successfully by the transition from the chaotic state to a large-scale periodic state. (4) Use noise to enhance the amplitude of weak failure signal. Yang and Cao [14] uses the principle of primary resonance and stochastic resonance to study time delay-controlled SD oscillator with stiffness nonlinearities, and it shows that the weak harmonic signal can be enhanced with appropriate noise and time delay. (5) In recent years, detecting weak signals based on the deep network has become a new way in the background of machine learning artificial intelligence that can automatically extract bearing signals and identify faults $[15,16]$. Xia et al. [17] propose an ensemble framework based on convolutional bidirectional long short-term memory with multiple time windows (MTW CNN-BLSTM ensemble) for accurately predicting remaining useful life.

It is worth noting that stochastic resonance is a nonlinear system involving noise and weak periodic signals; under the right conditions, the noise enhances the periodic signal of the weak signal where Kramers rate [18] is the key. And, the chaotic threshold is of great importance for detecting harmonic weak signal in the way of transition from the chaos to large-scale period, where the method of Melnikov function [19] can solve this problem very well. Moreover, a mass of data is needed to train the network layer with deep network detecting weak signals. In addition, Dong et al. [20] integrate a Bayesian updating prognostic model using sensor-based degradation information for computing each machine's time-to-failure (TTFs), with an opportunistic maintenance policy handling flexible system structures for optimizing the maintenance scheduling. In this paper, a new method for detecting weak signals is proposed based on SDDEs.

In the time domain, the basis of detecting weak signals is parameter identification, starting from the 1970s, showing a variety of system parameter recognition methods. Nayfeh [21] proposed a nonlinear system parameter recognition method, which is based on the response of nonlinear systems and the comparison between the system hypothesis model.

A method of recognition parameters based on the wavelet transform principle is proposed by Staszewski [22], which can identify parameters in multi-DOF nonlinear systems, but this method can only deal with free vibration system. Chen et al. [23] carried out parameter identification of nonlinear systems based on neural networks. In recent years, parameter identification is applied in various fields, such as automatic identification and medical research [24-26].

The incremental harmonic balance (IHB) method was originally developed by Lau et al. [27] for treating periodic structural vibrations and which is a semianalytical method; moreover, it is remarkably effective in computer implementation for obtaining both the stable and unstable solutions. Then, through the efforts of many scholars, the IHB method has been successfully applied to many fields of nonlinear vibration, and Shen [28] successfully applied the IHB method to study the bifurcation and the route-to-chaos analyses. Dou and Ye $[29,30]$ raised the method of IHB to rectify the harmonic coefficients in frequency response calculating, which may help the identification algorithm to correct the noise-induced distortion in the experiment, and compared with the harmonic balance (HB) method, the accuracy of parameter identification has obvious advantages. Moreover, these methods are applied in ordinary differential equations (ODEs), and it is necessary to study identifying parameters with the delay differential equations (DDEs). The study of time-delay system is more consistent with the real vibration because a state in the past will affect the vibration state now [31]. Recently, Zhang [32] applies the IHB method to solve the superharmonic resonance solution of nonlinear time-delay differential equations. The research methods of parameter estimation in time-delay chaotic systems are endless, for example, neural network method [33] and selfsynchronization method [34].

In this paper, for the first time, the IHB method is applied to SDDEs for the identification of weak signals, and from the result of parameter identification, it is more direct than the chaos detection method. We take stochastic delay Van der Pol-Duffing equation, for example, to identify parameters. The delay $\tau$ is reduced by scaling, and the results show that it does not affect the identification. Calculating the explicit expressions $K, P$, and $R$ is the important point of this paper, and then the explicit expressions of $K, P$, and $R$ are substituted into equation (9) and iterated until the $\|R\|$ is small enough; then, the result is the value of the parameter to be identified, which is clearly expressed. Finally, the ability of stochastic delay Van der Pol-Duffing system to detect weak signals is evaluated through the signal to noise ratio (SNR).

\section{Stochastic Van der Pol-Duffing Oscillator under Time-Delayed Feedback}

The Van der Pol-Duffing oscillator is a representative kind of system in the nonlinear system, and the system can show rich nonlinear dynamic behaviour with the change in strength in the period force $[19,35,36]$. As a classic chaotic system, Van der Pol-Duffing oscillator is often used in the modeling of dynamical systems; nowadays, there are many nonlinear problems in the fields of material resources, biology, engineering, psychiatry, and economics, which can be discussed and analyzed based on the modeling of nonlinear systems [37]. With the further study of vibration equation and the process of modeling, the stochastic Van der Pol-Duffing equation under the time-delayed feedback is more consistent with the process of actual measurement and signal acquisition, which has rich dynamic behaviour. The characteristic of being sensitive to a weak signal with strong noise perturbation makes it possible to be used in the field of weak signal detection. For stochastic Van der Pol oscillator with time-delayed feedback, some scholars have studied its response and stability analysis [38].

The stochastic Van der Pol-Duffing oscillator under time-delayed feedback is in the form of the following:

$$
\ddot{x}(t)+\alpha\left(x^{2}(t-\tau)-1\right) \dot{x}(t)+\beta x(t)=f \cos (\Omega t)+\sigma \xi(t),
$$


where $\alpha$ and $\beta$ are the damping coefficient and stiffness coefficient, respectively; $f \cos (\Omega t)$ is the external excitation; $f$ and $\Omega$ are the amplitude and angular frequency of external excitation, respectively; $\tau$ is the time delay; $x^{2}(t-\tau) \dot{x}(t)$ indicates that the square of displacement at $t-\tau$ will affect the velocity at $t$, namely, displacement feedback; and $\xi(t)$ is the Gaussian white noise with a constant power spectral density $\widetilde{K}$ and autocorrelation function $R(v)=E[\xi(t) \xi$ $(t+v)]=\delta(v)$, where $v$ is autocorrelation time and $\delta$ is Diarc function; moreover, $E[\cdot]$ denotes the expectation operator and $\sigma=\sqrt{2 \pi \widetilde{K}}$ denotes noise intensity.

For various parameters $f, \tau$, and $\Omega$, the system has rich nonlinear dynamic behaviours, seen from Figures 1-3.

\section{The Process of Parameter Identification}

The influence of noise on the result of parameter identification can be fully considered in the process of numerical simulation. We can write equation (1) without noise as the equation of state, as follows:

$$
\left\{\begin{array}{l}
\dot{y}_{1}(t)=y_{2}(t), \\
\dot{y}_{2}(t)=f \cos (\Omega t)-\alpha y_{1}^{2}(t-\tau) y_{2}(t)+\alpha y_{2}(t)-\beta y_{1}(t) .
\end{array}\right.
$$

Then, take scale transformation $t \longrightarrow(t / \tau)$ and write it as follows for convenience:

$$
\ddot{y}(t)-\alpha \tau^{2} \dot{y}(t)+\beta \tau^{2} y(t)+\sum_{i=1}^{M} b_{i} g_{i}(y(t), \dot{y}(t), y(t-1), \tau, t)=0,
$$

where $\quad M=2, b_{1}=\tau^{2} \alpha, b_{2}=-\tau^{2} f, g_{1}=y^{2}(t-1) \quad \dot{y}(t)$, $g_{2}=\cos (\Omega \tau t)$, and $b_{1} b_{2}$ are nonlinear parameters to be identified.

\section{Construct Increment Process}

$y, y(t-1)$, and $\left\{b_{i} \mid i=1,2\right\}$ are as follows:

$$
\begin{aligned}
y(t) & =y_{0}(t)+\Delta y(t), \\
y(t-1) & =y_{0}(t-1)+\Delta y(t-1), \\
b_{i} & =b_{i 0}+\Delta b_{i}, \quad i=1,2 .
\end{aligned}
$$

Working with initial values $\left\{b_{i 0}=0 \mid i=1,2\right\}$. Determine the initial values of the displacement $y_{0}(t)$ and $y_{0}(t-1)$ based on the experimental data, that is, depending on the displacement response on the $\left[t_{0}, t_{0}+T\right]$ when the system enters the steady-state period. Let $\omega=\Omega / \mu$ ( $\mu$ is an integer), period $T=2 \pi / \omega$. The initial values of displacement are expanded into the Fourier series in plural form, and they are written in the following matrix form, where $N$ is the degree of expansion of harmonic term:

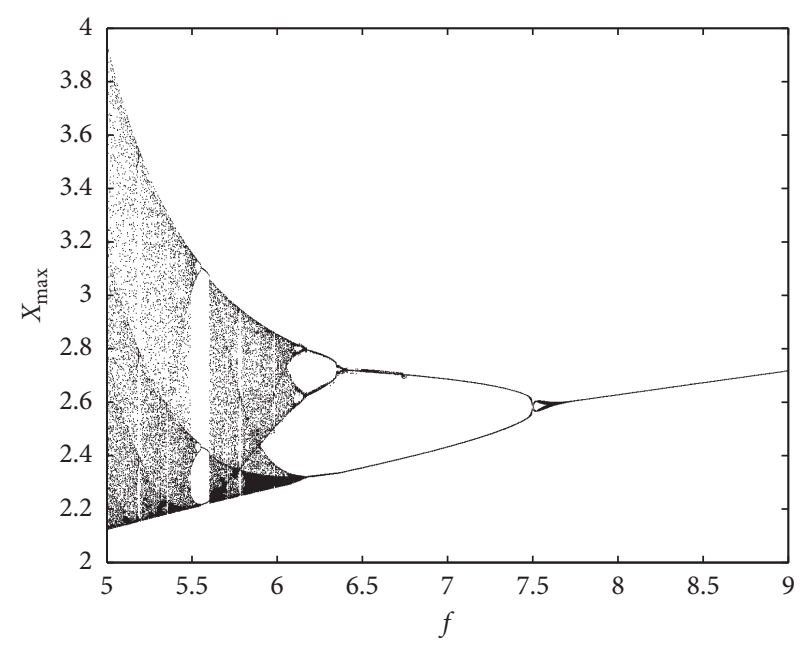

FIgURE 1: The bifurcation diagram of $f$ with certain fixed parameters $\tau=2, \alpha=5, \beta=1$, and $\Omega=1$ shows that chaotic state $\longrightarrow$ quasiperiodic motion $\longrightarrow$ period-doubling bifurcation $\longrightarrow$ periodic motion with the increasing $f$.

$$
\begin{gathered}
y_{0}(t)=\sum_{k=-N}^{N} e^{j k \omega t} \cdot a_{k}=Y_{1} \cdot A_{0}, \\
y_{0}(t-1)=\sum_{k=-N}^{N} e^{j k \omega(t-1)} \cdot a_{k}=Y_{2} \cdot A_{0},
\end{gathered}
$$

where

$$
\begin{aligned}
& Y_{1}=\left[e^{j(-N) \omega t} \ldots 1 \ldots e^{j N \omega t}\right], \\
& Y_{2}=\left[e^{j(-N) \omega(t-1)} \ldots 1 \ldots e^{j N \omega(t-1)}\right], \\
& A_{0}=\left[a_{-N} \ldots a_{0} \ldots a_{N}\right]^{T} .
\end{aligned}
$$

Each element in $A_{0}$ is the Fourier coefficient of each order, where the $k$-order Fourier coefficient is obtained by numerical integral transformation of the data series of the displacement response:

$$
\begin{aligned}
& a_{k 1}=\frac{1}{T} \int_{0}^{T} \tilde{y}_{0}(t) \cdot e^{-j k \omega t} \mathrm{~d} t, \\
& a_{k 2}=\frac{1}{T} \int_{0}^{T} \tilde{y}_{0}(t-1) \cdot e^{-j k \omega(t-1)} \mathrm{d} t,
\end{aligned}
$$

where $\tilde{y}_{0}(t)$ and $\tilde{y}_{0}(t-1)$ are a sequence of displacement response measured and collected in experiments. To the extent that the error allows $a_{k 1} \approx a_{k 2}$, $a_{k}=(1 / T) \int_{0}^{T} \tilde{y}_{0}(t) \cdot e^{-j k \omega t} \mathrm{~d} t$. Working with the incremental processes. Write $\Delta y(t)$ and $\Delta y(t-1)$ in the form of Fourier series in the plural form: 


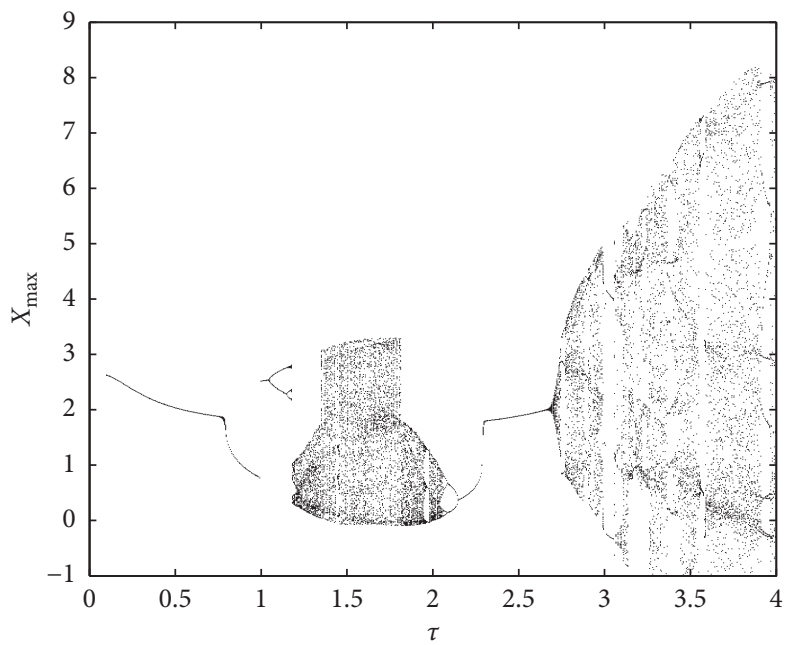

FIgURE 2: The bifurcation diagram of $\tau$ with certain fixed parameters $\alpha=0.5, \beta=1, \Omega=1$, and $f=1$.

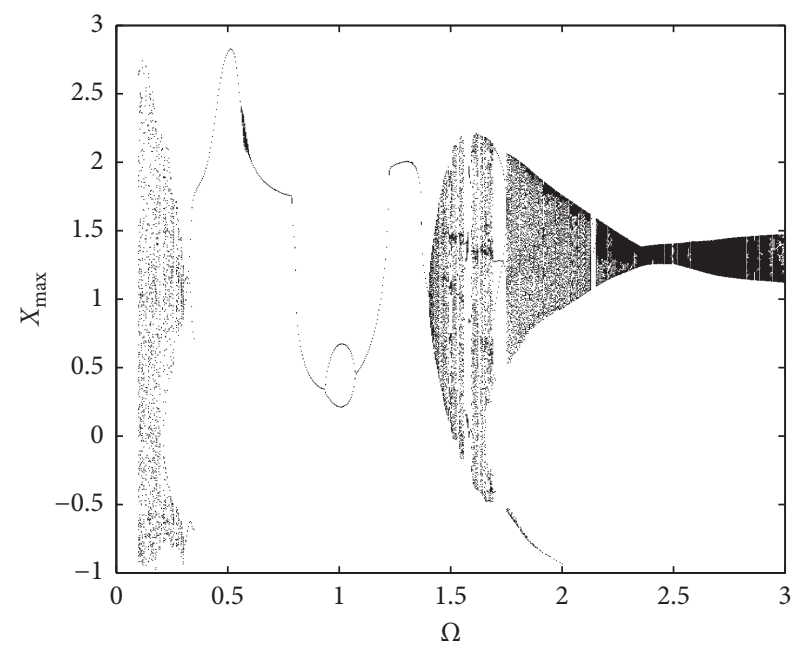

Figure 3: The bifurcation diagram of $\Omega$ with certain fixed parameters $\alpha=0.45, \beta=1, \tau=2$, and $f=1$.

$$
\begin{aligned}
\Delta y(t) & =Y_{1} \cdot \Delta A, \\
\Delta y(t-1) & =Y_{2} \cdot \Delta A, \\
\Delta A & =\left[\Delta a_{-N} \ldots \Delta a_{0} \ldots a_{N}\right]^{T} .
\end{aligned}
$$

The increments of the parameters are $\Delta b=\left[\begin{array}{ll}\Delta b_{1} & \Delta b_{2}\end{array}\right]^{T}$.

Formulas in (4) are substituted into equation (3), by the means of Taylor unfold, and omitting the incremental two times and more than two high-order items, we can obtain

$$
\begin{aligned}
& \Delta \ddot{y}(t)-\alpha \tau^{2} \Delta \dot{y}(t)+\beta \tau^{2} \Delta y(t)+2 b_{10} y_{0}(t-1) \dot{y}_{0}(t) \Delta y(t-1) \\
& +b_{10} y_{0}^{2}(t-1) \Delta \dot{y}(t)+y_{0}^{2}(t-1) \dot{y}(t) \Delta b_{1}+\cos (\Omega \tau t) \Delta b_{2} \\
& =-\ddot{y}_{0}(t)+\alpha \tau \dot{y}_{0}(t)-\beta \tau^{2} y_{0}(t)-b_{10} y_{0}^{2}(t-1) \dot{y}_{0}(t)-b_{20} \cos (\Omega \tau t) .
\end{aligned}
$$

\section{Construct Increment Equation}

With the method of Galerkin and the regular $\Delta y(t)=Y_{1} \Delta A=\Delta A^{T} Y_{1}^{T}=\left(\Delta A Y_{1}\right)^{T}=(\Delta y(t))^{T}$, we can obtain

$$
\begin{aligned}
& (\delta \Delta A)^{T} \int_{t_{0}}^{t_{0}+T} Y_{1}^{T}\left[\ddot{Y}_{1}-\alpha \tau \dot{Y}_{1}+\beta \tau^{2} Y_{1}+2 b_{10} y_{0}(t-1) \dot{y}_{0}(t) Y_{2}\right. \\
& \left.\quad+b_{10} y_{0}^{2}(t-1) \dot{Y}_{1}\right] \mathrm{d} t \cdot \Delta A \\
& \quad+(\delta \Delta A)^{T} \int_{t_{0}}^{t_{0}+T} Y_{1}^{T}\left[y_{0}^{2}(t-1) \dot{y}(t)\right] d t \cdot \Delta b_{1} \\
& \quad+(\delta \Delta A)^{T} \int_{t_{0}}^{t_{0}+T} Y_{1}^{T}[\cos (\Omega \tau t)] \mathrm{d} t \cdot \Delta b_{2} \\
& \quad=(\delta \Delta A)^{T} \int_{t_{0}}^{t_{0}+T} Y_{1}^{T}\left[-\ddot{y}_{0}(t)+\alpha \tau \dot{y}_{0}(t)-\beta \tau^{2} y_{0}(t)\right. \\
& \left.\quad-b_{10} y_{0}^{2}(t-1) \dot{y}_{0}(t)-b_{20} \cos (\Omega \tau t)\right] \mathrm{d} t .
\end{aligned}
$$

With arbitrary of $\delta \Delta A$, we can obtain the incremental equation as follows:

$$
K \Delta A+P \Delta b=R .
$$

The elements of $K, P$, and $R$ are

$$
\begin{aligned}
K= & \frac{1}{T} \int_{t_{0}}^{t_{0}+T} Y_{1}^{T}\left[\ddot{Y}_{1}-\alpha \tau \dot{Y}_{1}+\beta \tau^{2} Y_{1}+2 b_{10} y_{0}(t-1) \dot{y}_{0}(t) Y_{2}\right. \\
& \left.+b_{10} y_{0}^{2}(t-1) \dot{Y}_{1}\right] \mathrm{d} t \\
P_{1}= & \frac{1}{T} \int_{t_{0}}^{t_{0}+T} Y_{1}^{T}\left[y_{0}^{2}(t-1) \dot{y}(t)\right] d t, \quad P_{2}=\frac{1}{T} Y_{1}^{T}[\cos (\Omega \tau t)] \mathrm{d} t \\
R= & \frac{1}{T} \int_{t_{0}}^{t_{0}+T} Y_{1}^{T}\left[-\ddot{y}_{0}(t)+\alpha \tau \dot{y}_{0}(t)-\beta \tau^{2} y_{0}(t)\right. \\
& \left.-b_{10} y_{0}^{2}(t-1) \dot{y}_{0}(t)-b_{20} \cos (\Omega \tau t)\right] \mathrm{d} t .
\end{aligned}
$$


TABLE 1: Parameter recognition results of incremental harmonic equilibrium method in different states of time-delay system, where $b_{1}^{*}=\alpha \tau$ and $b_{2}^{*}=-\tau^{2} f$ are exact values.

\begin{tabular}{ccccccccccc}
\hline \multirow{2}{*}{ Parameters } & $\sigma$ & \multicolumn{3}{c}{$0 \%$} & \multicolumn{3}{c}{$1 \%$} & & \multicolumn{2}{c}{$5 \%$} \\
& $N$ & $f=6$ & $f=7$ & $f=8$ & $f=6$ & $f=7$ & $f=8$ & $f=6$ & $f=7$ \\
\hline & 10 & 10.00968 & 10.00962 & 10.00515 & 10.00962 & 10.00962 & 10.00512 & 10.00975 & 10.00965 & 10.00515 \\
$b_{1}$ & 15 & 10.00956 & 10.00957 & 10.00497 & 10.00961 & 10.00951 & 10.00511 & 10.00957 & 10.00950 & 10.00503 \\
& 20 & 10.00959 & 10.00954 & 10.00503 & 10.00961 & 10.00951 & 10.00506 & 10.00972 & 10.00952 & 10.00503 \\
& 25 & 10.00961 & 10.00951 & 10.00501 & 10.00957 & 10.00950 & 10.00510 & 10.00963 & 10.00954 & 10.00502 \\
& 30 & 10.00959 & 10.00949 & 10.00503 & 10.00973 & 10.00950 & 10.00500 & 10.00951 & 10.00963 & 10.00501 \\
\hline & 10 & 24.00924 & 28.00882 & 32.00723 & 24.00920 & 28.00860 & 32.00723 & 24.00903 & 28.00873 & 32.00723 \\
$b_{2}$ & 15 & 24.00941 & 28.00886 & 32.00769 & 24.00950 & 28.00871 & 32.00710 & 24.00933 & 28.00871 & 32.00810 \\
& 20 & 24.00937 & 28.00889 & 32.00769 & 24.00934 & 28.00882 & 32.00820 & 24.00932 & 28.00872 & 32.00841 \\
& 25 & 24.00940 & 28.00882 & 32.00774 & 24.00931 & 28.00881 & 32.00720 & 24.00891 & 28.00881 & 32.00790 \\
& 30 & 24.00932 & 28.00886 & 32.00769 & 24.00943 & 28.00881 & 32.00770 & 24.00937 & 28.00877 & 32.00757 \\
\hline
\end{tabular}
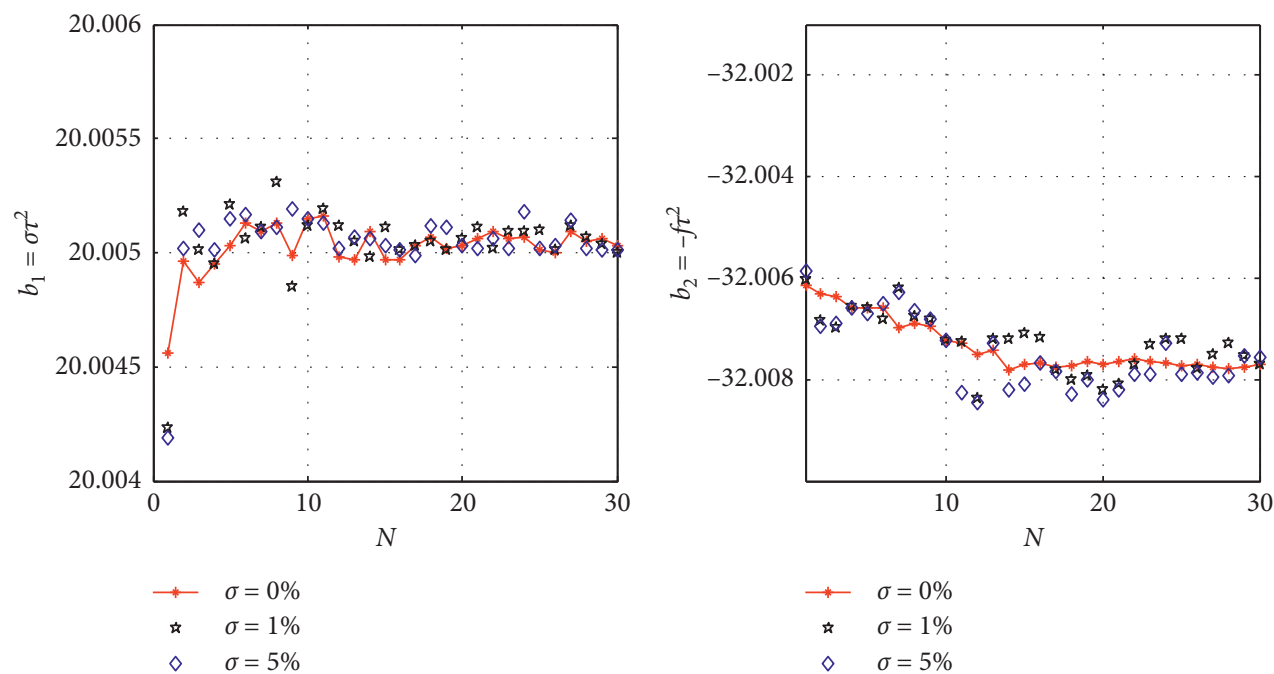

FIgURE 4: The influence of noise on parameter recognition in a periodic state with $f=8$ and $\tau=2$.
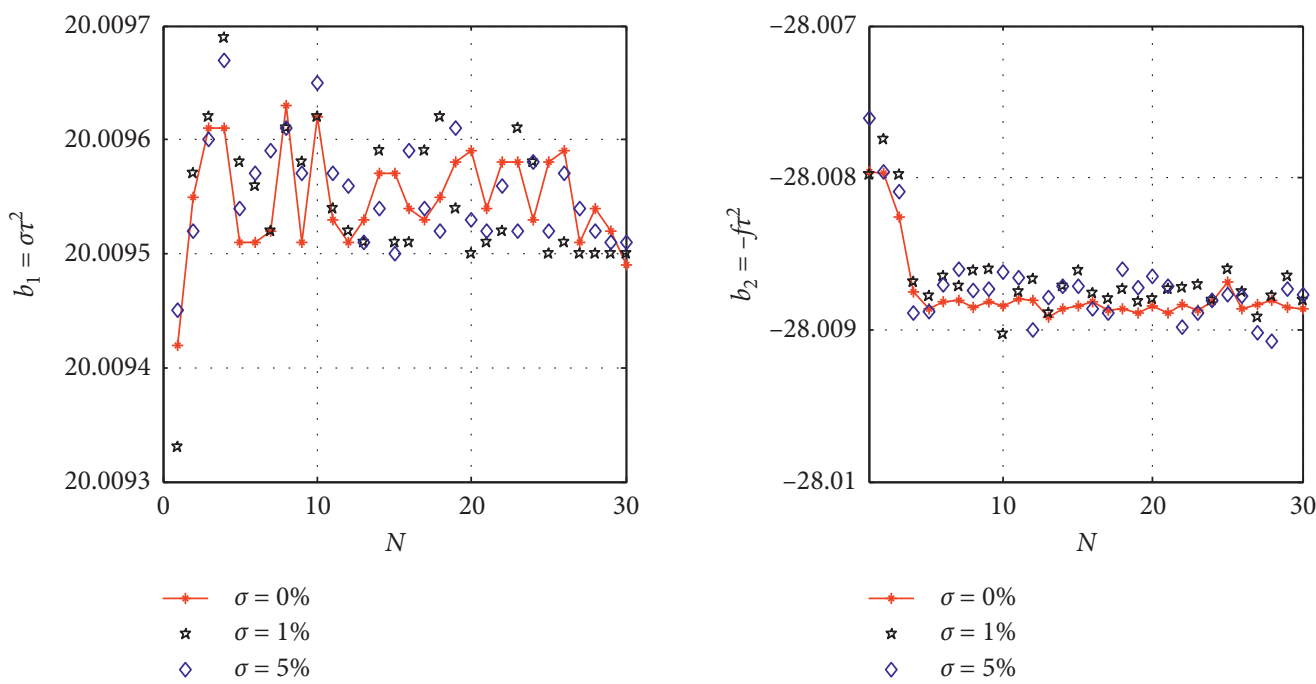

FIGURE 5: The influence of noise on parameter recognition in period-2 state with $f=7$ and $\tau=2$. 

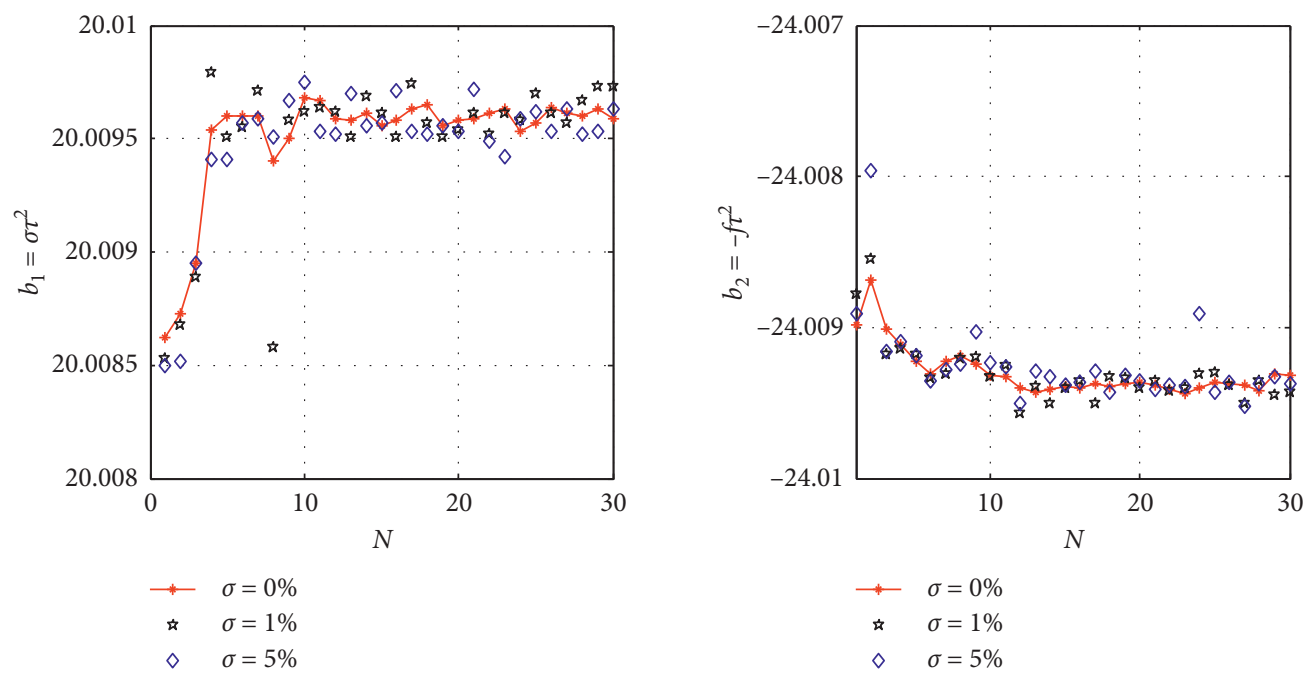

FIGURE 6: The influence of noise on parameter recognition in a chaotic state with $f=6$ and $\tau=2$.

To know the explicit expression of elements in $K, P$, and $R$, according to the plural form of Fourier series of the initial displacement expansion, the coefficients of the Fourier series in the plural form corresponding to $\dot{y}_{0}(t), y_{0}{ }^{2}(t-1), y_{0}{ }^{2}(t-1) \dot{y}_{0}(t), \ddot{y}_{0}(t), \cos (\Omega t) \quad$ are obtained:

$$
\begin{aligned}
& \dot{y}_{0}(t)=\sum_{k=-N}^{N} a_{k 3} \cdot e^{j k \omega t}, \quad a_{k 3}=a_{m} \cdot j m \omega \delta_{m}^{k}, \\
& y_{0}^{2}(t-1)=\sum_{k=-N}^{N} a_{k 4} \cdot e^{j k \omega t}, \quad a_{k 4}=a_{m} a_{l} \cdot e^{-j \omega(m+l)} \delta_{m+l}^{k}, \\
& y_{0}(t-1) \dot{y}_{0}(t)=\sum_{k=-N}^{N} a_{k 5} \cdot e^{j k \omega t}, \quad a_{k 5}=a_{m} a_{n 3} e^{-j m \omega} \delta_{m+n}^{k}, \\
& y_{0}^{2}(t-1) \dot{y}_{0}(t)=\sum_{k=-N}^{N} a_{k 6} \cdot e^{j k \omega t}, \quad a_{k 6}=a_{m 4} a_{l 3} \delta_{m+l}^{k}, \\
& \ddot{y}_{0}(t)=\sum_{k=-N}^{N} a_{k 7} \cdot e^{j k \omega t}, \quad a_{k 7}=-m^{2} \omega^{2} a_{m} \delta_{m}^{k} .
\end{aligned}
$$

Calculate the Fourier coefficient $q_{k}$ of the harmonic excitation item $\cos (\Omega \tau t)$ based on the experimental data:

$$
\cos (\Omega \tau t)=\sum_{k=-N}^{N} q_{k} \cdot e^{j k \omega t}, \quad q_{k}=\frac{1}{T} \int_{0}^{T} \frac{\widetilde{F}(t)}{\rho} e^{-j k \omega t} \mathrm{~d} t,
$$

where $\widetilde{F}(t)$ is the excitation force signal collected experimentally and $\rho$ is shaking force amplitude. Let $\bar{m}=m+N+1, \bar{n}=n+N+1$, then we can write the explicit expression of elements in $K, P$, and $R$ :

$$
\begin{aligned}
K(\bar{m}, \bar{n})= & \left(-n^{2} \omega^{2}-\alpha \tau j n \omega+\beta \tau^{2}\right) \delta_{m+n}^{0} \\
& +\sum_{k=-N}^{N}\left(2 b_{10} a_{k 2}+b_{10} a_{k 3} j n \omega\right) e^{-j n \omega} \delta_{m+n+k}^{0}, \\
P_{1}(\bar{m}, 1)= & a_{k 4} \delta_{m+k}^{0} \quad P_{2}(\bar{m}, 2)=q_{k} \delta_{m+k}^{0}, \\
R(\bar{m}, 1)= & \left(-a_{k 5}+\alpha \tau a_{k 1}-\beta \tau^{2} a_{k}-b_{10} a_{k 4}-b_{20} q_{k}\right) \delta_{m+k}^{0} .
\end{aligned}
$$

Equation (16) is substituted into equation (12) iteratively. It is clear that equation (12) has $2 N+1+2$ unknowns $\left\{\Delta a_{k}, k=-N, \ldots, N ; \Delta b_{1}, \Delta b_{2}\right\}$, but there are $2 N+1$ equations. So the increment of the harmonic item coefficients $\Delta A$ and $\Delta b$ as the tuning amount is obtained by equation (12) on condition that no less than 2 principal harmonic coefficients increment $\Delta a_{k}$ zero. $\Delta y, y$, and $b_{1}, b_{2}$ can be obtained from equations (4) and (9), respectively, and then replace the initial values of $y_{0}$ and $b_{10}, b_{20}$ to (16) and enter the next iteration, and so on. We do not stop iterating until $\|R\|$ is small enough. When the iteration stops, the results of $b_{1}$ and $b_{2}$ are the value of the parameter to be identified.

\section{The Results of Identification}

Consider the parameters $b_{1}=\alpha \tau^{2}$ and $b_{2}=-f \tau^{2}$ to be identified, and given parameter initial value $b_{10}=b_{20}=0$; constructing incremental equations, select $a_{0}, a_{+1}, a_{+2}$ as the main harmonic coefficients, that is, $\Delta a_{0}=\Delta a_{ \pm 1}=\Delta a_{ \pm 2}=0$; solve the remaining harmonic item increments and parameter increments. and iterate over and over until the algorithm converges steadily. The identification results are shown below (Table 1). 
TABLE 2: Results of parameters identification with the method of IHB.

\begin{tabular}{llccr}
\hline \multirow{2}{*}{ Parameter } & $\sigma$ & $0 \%$ & \multicolumn{2}{c}{$\begin{array}{c}1 \% \\
\end{array}$} \\
& $N$ & & -32.020633 & -32.020623 \\
& 10 & -32.020632 & -32.020637 & -32.020638 \\
$\left(b^{*}=-\tau^{2}(f+r)\right)$ & 15 & -32.020632 & -32.020643 & -32.020641 \\
& 20 & -32.020633 & -32.020631 & -32.020630 \\
& 25 & -32.020641 & -32.020620 & -32.020625 \\
\hline
\end{tabular}

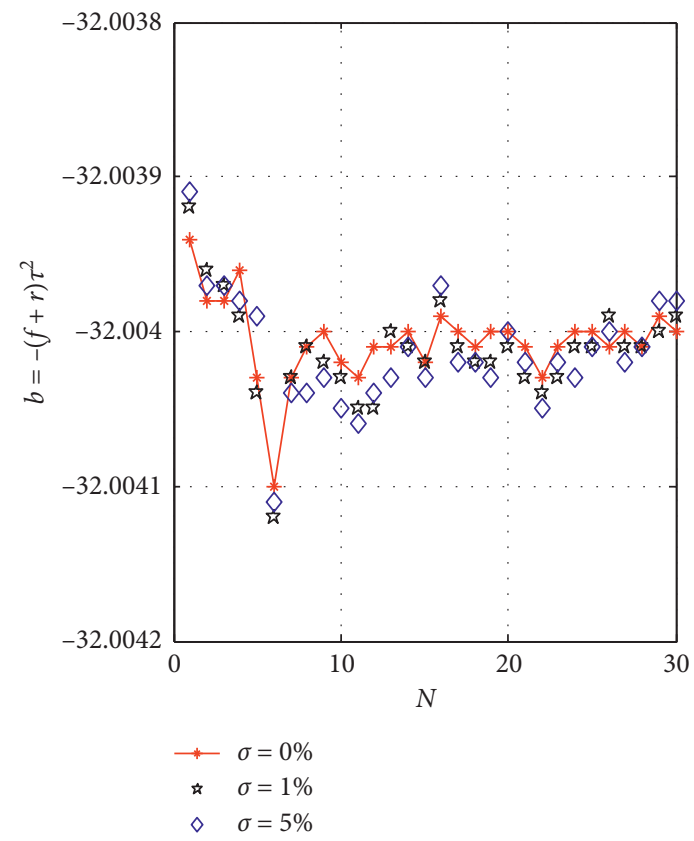

Figure 7: The influence of noise on parameter recognition with fixed parameters $f=8, r=0.001, \alpha=5, \beta=1, \Omega=1$, and $\tau=2$.
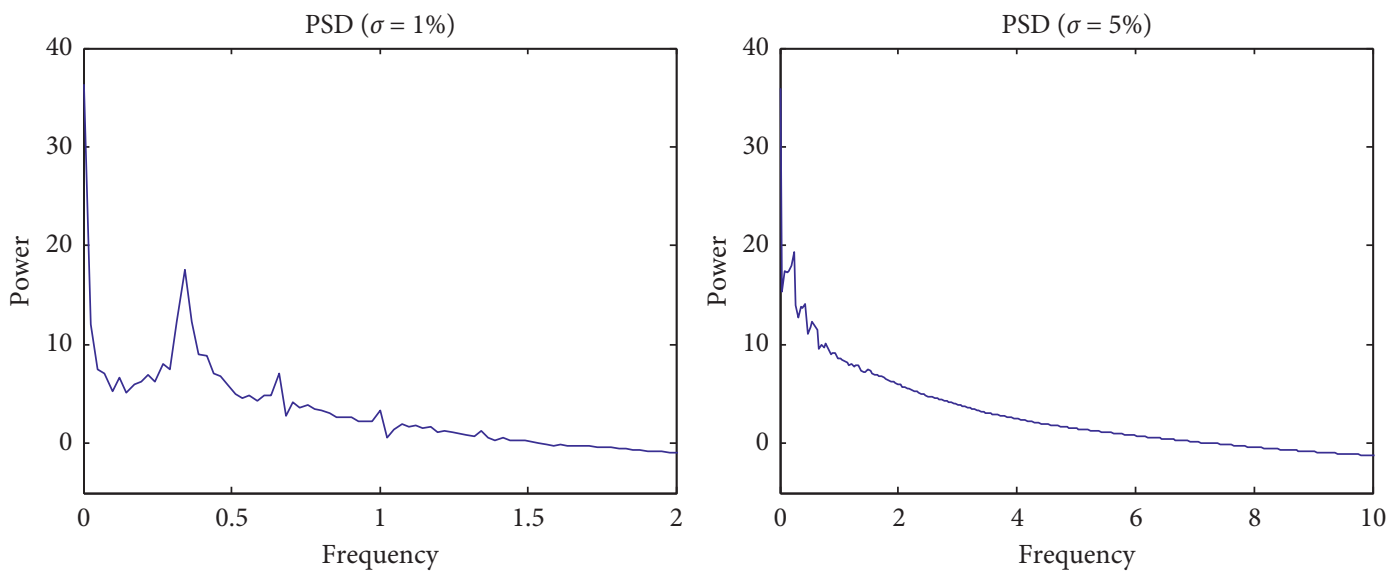

FIGURE 8: PSD under different noise intensity $\sigma=1 \%$ and $\sigma=5 \%$.

Figures 4-6 show that the recognition results of parameters $b_{1}$ and $b_{2}$ when the system is in different states, such as period, period-2, and chaos. We can get from the figures that the results of parameter recognition are stable when the system is in different states. It is worth noting that the difficulty of parameter recognition using the IHB method is finding the appropriate period initial displacement value from the steady-state response to recognize the parameters. 


\section{Identify the Amplitude of Weak Signals}

Add the weak signal under strong noise $r \tau^{2} \cos (\Omega \tau t)+\sigma \tau^{2} \xi(t)$ to equation (3) with $r=0.001$ and $\tau=2$, and we can obtain

$$
\begin{aligned}
\ddot{y}- & \alpha \tau^{2} \dot{y}(t)+\beta \tau^{2} y(t)+\alpha \tau^{2}\left(y^{2}(t-1)\right) \dot{y}(t) \\
& =(f+r) \tau^{2} \cos (\Omega \tau t)+\sigma \tau^{2} \xi(t),
\end{aligned}
$$

where the parameter to be identified is $b=-(f+r) \tau 2$. From the results of identifying Table 2 and Figure 7 with certain parameters, we can obtain that $b \approx-32.004$ and then the amplitude of the weak harmonic signal is $r=0.001$, which is consistent with the added signal amplitude so that the weak signal can be identified well.

Consider the SNR to judge how well the system detects weak signal under the noise intensity $\sigma=0.05$ :

$$
S N R=10 \lg \left(0.5 \frac{r^{2}}{\sigma^{2}}\right)=10 \lg \left(0.5 \frac{0.001^{2}}{0.05^{2}}\right)=-36.99 \mathrm{~d} B
$$

The amplitude of the weak signal can be detected under the condition of low SNR, and it is proved that this method is worthy of application and popularization in the field of DDEs.

According to the system response power spectral density function under different noises, we can obtain the power spectral density (PSD) images, as shown in Figure 8; the frequency at the highest energy is $f r e=(1 / T)=0.3180$ obviously. The angular frequency of a weak signal is $\Omega \tau=(2 \pi / T)$, so $T=\pi$, and then, the frequency of a weak signal is $(1 / T)=0.3182$. We can detect weak signals from strong noise.

\section{Conclusions}

In this paper, there is a rich dynamic behaviour of the stochastic Van der Pol-Duffing oscillator with noise interference, and the parameters can be identified by the method of IHB in different states. For a very low SNR, the detection results are very good and this method can be extended to detect weak signals in nonlinear DDEs containing the certain noise.

\section{Data Availability}

No data were used to support this study.

\section{Conflicts of Interest}

The authors declare that there are no conflicts of interest regarding the publication of this paper.

\section{Acknowledgments}

This work was supported by the Natural Science Foundation of China (nos. 11602151 and 11872253), the Natural Science Foundation for Outstanding Young Researcher in Hebei Province of China (no. A2017210177), and the Foundation of Hebei Education Department of China (Grant no. QN2018050).

\section{References}

[1] T. Kapitaniak, Chaotic Oscillators: Theory and Applications, World Scientific, Singapore, 1992.

[2] P. D. McFadden and J. D. Smith, "Vibration monitoring of rolling element bearings by the high-frequency resonance technique - a review," Tribology International, vol. 17, no. 1, pp. 3-10, 1984.

[3] S. S. Dave, Vibration Analysis for Electronic Equipment, Wiley-Blackwell, Hoboken, NJ, USA, 2000.

[4] N. Fantuzzi, F. Tornabene, M. Bacciocchi, and R. Dimitri, "Free vibration analysis of arbitrarily shaped functionally graded carbon nanotube-reinforced plates," Composites Part B: Engineering, vol. 115, pp. 384-408, 2017.

[5] F. M. Atay, "Van der pol's oscillator under delayed feedback," Journal of Sound and Vibration, vol. 218, no. 2, pp. 333-339, 1998.

[6] Z. H. Lai and Y. G. Leng, "Generalized parameter adjusted stochastic resonance of duffing oscillator and its application to weak signal detection," Sensors, vol. 15, no. 9, pp. 2132721349, 2015.

[7] D. Butusov, T. Karimov, A. Voznesenskiy, D. Kaplun, V. Andreev, and V. Ostrovskii, "Filtering techniques for chaotic signal processing," Electronics, vol. 7, no. 12, p. 450, 2018.

[8] V. Purushotham, S. Narayanan, and S. A. N. Prasad, "Multifault diagnosis of rolling bearing elements using wavelet analysis and hidden Markov model based fault recognition," NDT \& E International, vol. 38, no. 8, pp. 654-664, 2005.

[9] B. Pang, G. Tang, T. Tian, and C. Zhou, "Rolling bearing fault diagnosis based on an improved HTT transform," Sensors, vol. 18 , no. 4, p. 1203, 2018.

[10] H. Qiu, J. Lee, J. Lin, and G. Yu, "Wavelet filter-based weak signature detection method and its application on rolling element bearing prognostics," Journal of Sound and Vibration, vol. 289, no. 4-5, pp. 1066-1090, 2006.

[11] D. L. Donoho, "De-noising by soft-thresholding," IEEE Transactions on Information Theory, vol. 41, no. 3, pp. 613627, 1995.

[12] M. E. Kounavis, "Image de-noising using an equalized gradient space," US 10262 397B2, April 2019.

[13] Z. Zhao and S. Yang, "Application of van der polduffing oscillator in weak signal detection," Computers \& Electrical Engineering, vol. 41, no. 1-8, 2015.

[14] T. Yang and Q. Cao, "Delay-controlled primary and stochastic resonances of the sd oscillator with stiffness nonlinearities," Mechanical Systems and Signal Processing, vol. 103, pp. 216235, 2018

[15] R. Zhao, R. Yan, Z. Chen, K. Mao, P. Wang, and R. X. Gao, "Deep learning and its applications to machine health monitoring," Mechanical Systems and Signal Processing, vol. 115, pp. 213-237, 2019.

[16] Y. Lei, N. Li, L. Guo, N. Li, T. Yan, and J. Lin, "Machinery health prognostics: a systematic review from data acquisition to rul prediction," Mechanical Systems and Signal Processing, vol. 104, pp. 799-834, 2018.

[17] T. Xia, Y. Song, Y. Zheng, E. Pan, and L. Xi, "An ensemble framework based on convolutional bi-directional LSTM with multiple time windows for remaining useful life estimation," Computers in Industry, vol. 115, p. 103182, 2020. 
[18] L. Gammaitoni, P. Hänggi, P. Jung, and F. Marchesoni, "Stochastic resonance," Reviews of Modern Physics, vol. 70, no. 1, pp. 223-287, 1998.

[19] R. Tian, X.-W. Yang, Q.-J. Cao, and Wu Qi-Liang, Bifurcations and chaotic threshold for a nonlinear system with an irrational restoring force," Chinese Physics B, vol. 21, no. 2, Article ID 020503, 2012.

[20] Y. Dong, T. Xia, X. Fang, Z. Zhang, and L. Xi, "Prognostic and health management for adaptive manufacturing systems with online sensors and flexible structures," Computers \& Industrial Engineering, vol. 133, pp. 57-68, 2019.

[21] A. H. Nayfeh, "Parametric identification of nonlinear dynamic systems," Computers \& Structures, vol. 20, no. 1-3, pp. 487-493, 1985.

[22] W. J. Staszewski, "Identification of non-linear systems using multi-scale ridges and skeletons of the wavelet transform," Journal of Sound and Vibration, vol. 214, no. 4, pp. 639-658, 1998.

[23] S. Chen, S. A. Billings, and P. M. Grant, "Non-linear system identification using neural networks," International Journal of Control, vol. 51, no. 6, pp. 1191-1214, 1990.

[24] L. Zhu, L. Chen, D. Zhao, J. Zhou, and W. Zhang, "Emotion recognition from Chinese speech for smart affective services using a combination of svm and dbn," Sensors, vol. 17, no. 7, p. 1694, 2017.

[25] J. Lee, L. Jin, D. Park, and Y. Chung, "Automatic recognition of aggressive behavior in pigs using a kinect depth sensor," Sensors, vol. 16, no. 5, p. 631, 2016.

[26] C.-C. Yang, Y.-L. Hsu, K.-S. Shih, and J.-M. Lu, "Real-time gait cycle parameter recognition using a wearable accelerometry system," Sensors, vol. 11, no. 8, pp. 7314-7326, 2011.

[27] S. L. Lau and Y. K. Cheung, "Amplitude incremental variational principle for nonlinear vibration of elastic systems," Journal of Applied Mechanics, vol. 48, no. 4, pp. 959-964, 1981.

[28] J. H. Shen, K. C. Lin, S. H. Chen, and K. Y. Sze, "Bifurcation and route-to-chaos analyses for Mathieu-Duffing oscillator by the incremental harmonic balance method," Nonlinear Dynamics, vol. 52, no. 4, pp. 403-414, 2008.

[29] S.-G. Dou and M. Ye, "Nonlinear identification in frequency domain for parametric excitation system based on harmonic balance principle," Journal of Vibration and Shock, vol. 28, no. 12, pp. 123-127, 2009.

[30] M. Ye, S. Dou, W. Zhang, and Z. Zeng, "Nonlinear identification of systems with parametric excitation," Science China Technological Sciences, vol. 54, no. 8, pp. 2080-2089, 2011.

[31] R. David Driver, Ordinary and Delay Differential Equations, Vol. 20, Springer Science \& Business Media, Berlin, Germany, 2012.

[32] H. Zhang, X. H. Wu, and L. Zhang, "Analysis of subharmonic resonance response of the time-delayed system," Advanced Materials Research, vol. 163-167, pp. 2910-2915, 2011.

[33] W. Lim, D. Jang, and T. Lee, "Speech emotion recognition using convolutional and recurrent neural networks," in Proceedings of the 2016 Asia- Pacific Signal and Information Processing Association Annual Summit and Conference (APSIPA), pp. 1-4, IEEE, Jeju, South Korea, December 2016.

[34] H.-T. Yau, S.-Y. Wu, C.-L. Chen, and Y.-C. Li, "Fractionalorder chaotic self-synchronization-based tracking faults diagnosis of ball bearing systems," IEEE Transactions on Industrial Electronics, vol. 63, no. 6, pp. 3824-3833, 2016.

[35] Z.-h. Lai and Y.-g. Leng, "Weak-signal detection based on the stochastic resonance of the bistable duffing oscillator and its application in incipient fault diagnosis," Mechanical Systems and Signal Processing, vol. 81, pp. 60-74, 2016.
[36] Z. Yang and H. Brunner, "Blow-up behavior of Hammersteintype delay Volterra integral equations," Frontiers of Mathematics in China, vol. 8, no. 2, pp. 261-280, 2013.

[37] M. Cooper, P. Heidlauf, and T. Sands, "Controlling chaosforced van der Pol equation," Mathematics, vol. 5, no. 4, p. 70, 2017.

[38] X. L. Yang and Z. K. Sun, "Research on parametric resonance in a stochastic van der Pol oscillator under multiple time delayed feedback control," International Journal of Non-linear Mechanics, vol. 45, no. 6, pp. 621-627, 2010. 